\title{
Persistent physics-based crisis management framework: A case study of traffic in the Nantes city due to flood exposure
}

\author{
Nafe Moradkhani \\ IMT Mines Albi, France \\ nafe.moradkhani@mines-albi.fr
}

\author{
Hélène Dolidon \\ CEREMA, Nantes, France \\ helene.dolidon@cerema.fr
}

\author{
Frederick Benaben \\ IMT Mines Albi, France \\ frederick.benaben@mines-albi.fr
}

\begin{abstract}
In the context of crisis, the characteristics of the crisis area and the operational measures of the community play key roles in managing the crisis. The Nantes ring road in France is always exposed to flooding and its disruptions. To anticipate the disruptions and timely preventive actions for this frequent phenomenon, the main challenges are (i) forecast of vehicles' flows, (ii) capacity of the ring road to handle the traffic (iii) evaluate the performance of alternate routes during the flooding. The flooded area as a system has components of (i) the flood (e.g. time of onset, magnitude, intensity, etc.), (ii) the area (e.g. geographical features, temporary perimeter barriers, dam, diversion canals), and (iii) the community (e.g. reaction time, emergency strain, evacuation delay). The chosen approach to conduct this anticipative study consists of collecting data about forecasts and using simulation models to work simultaneously on evaluating the performance of the ring road and its alternative routes.
\end{abstract}

\section{Introduction}

The city of Nantes is surrounded by the $43 \mathrm{~km}$ long Nantes Ring Road (RN 844). The ring road is operated by the Interdepartmental Direction of Western Roads (DIRO), except on a portion to the north that is operated by Cofiroute (see Fig. 1) [1].

With the development of the urban area, the traffic of the ring road has increased significantly and it reaches more than 100,000 vehicles per day in certain sections; therefore, the network congestion is notable at peak hours. Up to 100,000 vehicles circulate on the ring road with the traffic peaks in the morning ( 8 am-9 am) and evening (5 pm-6 pm) [1, 2, 3]. Significant flows are observed in the south-north (SN) direction in the morning and the opposite direction in the evening. Most of the jobs are located in the northern part of the city and are the main reason for the difference between these flows.

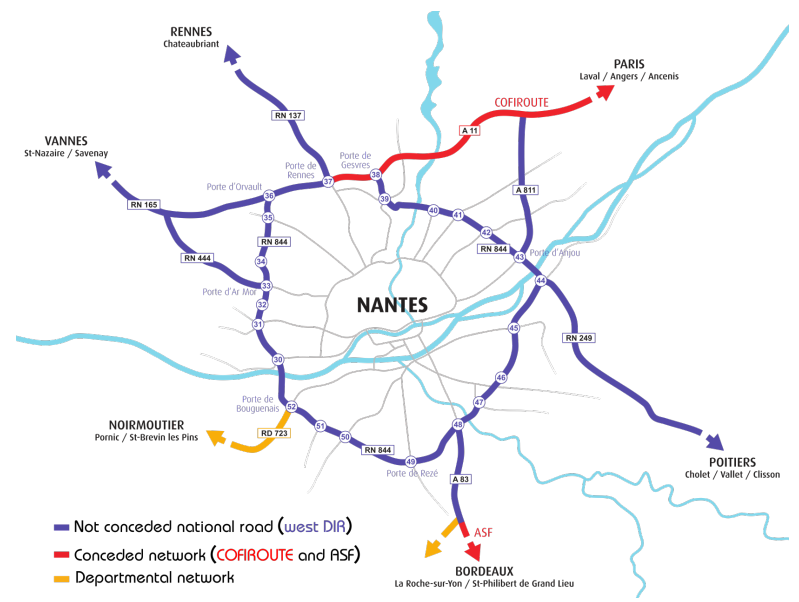

Figure 1: Nantes ring road and its operators.

The northeast part of the Nantes ring road is flooded frequently due to the overflow of the "Gesvres" river. The Gesvres river originates from the Erdre river which is started near Angers city and ends in the city of Nantes. The historical data from the Joneliere station which reports the Gesvres water level shows whenever the Erdre river overflows so does the Gesvres river [3]. This paper focuses on a predictive model of the traffic on the ring road during the flooding to cope with flooding disruptions [3, 4, 5].

Preventive systems with the ability to detect, predict, and make decisions are very useful in the field of crisis management. The necessity of predictor systems to deal with unforeseen events such as flooding is indispensable. These systems are able to detect crises as early as possible and implement appropriate solutions to manage them [6].

This paper relies on an intelligent physics-based framework introduced in [7] to manage crises. This original framework, Physics Of Decision (POD), considers that crisis can be seen as physical forces applied to the system which may push or pull it in its performance space by varying the system's KPIs (Key 
Performance Indicators) [8]. The framework guides decision-makers in assessing the risks that may happen to a system. Fig. 2 describes the framework, including its components and the relationships between them.

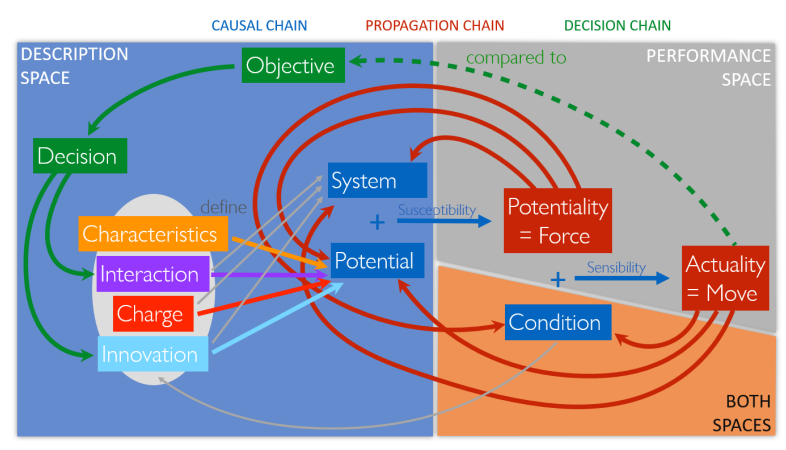

Figure 2: POD crisis management framework.

In the POD framework, the considered system is the Nantes ring road which is facing some potentials, e.g. a heavy traffic jam. These potentials include (i) Characteristics, i.e. the environmental potentials of the system such as precipitation which causes its nearby river overflow and road blockage or it might happen during holidays, (ii) Interactions, i.e. vehicle flows of the routes leading to the ring road, (iii) Charges, i.e. mandatory system costs including minimum trip time considering maximum speed allowed between two spots on the ring road at normal times/ peak times, and (iv) Innovations, i.e. some initiatives dedicated to modify or improve the structure or the behavior of the system such as partially activation some alternative routes in peak hours or totally during the flood. The susceptibility of the ring road to the traffic jam generates some potentialities (i.e. heavy traffic jam considered as physical forces). Heavy traffic jam as potential might be activated by the water level of the Gesvres river as the condition which triggers the forces and changes the system's performance: the potentialities become actualities and change the performance of the system. By comparing actualities to the actual objectives of the system (e.g. light traffic on the ring road), managers take some decisions to minimize the difference between the current state of the system and its objectives. A detailed definition of the components and their relationships is given in [9].

The main objective of this article is to introduce a data-driven simulation model and POD-based predictive crisis management system to control the traffic jam and to investigate its value using a case study from the Nantes ring road which is permanently exposed to the Erdre river flood.
The remainder of this paper is organized as follows: Section 2 highlights related existing research works and scientific contributions. Section 3 describes the Intelligent Crisis Management framework and its principles. Section 4 deals with the implementation through the simulation of the case study. Section 5 examines the results of the simulation-based investigation for the Nantes ring road. Finally, section 6 concludes this research work and provides avenues for further research.

\section{Background and related works}

Flooding in the Nantes ring road is a regular phenomenon due to the overflow of the Erdre river [10]. The height of the Gesvres river is the indicator of this crisis [11]. The huge traffic on the ring road in the flooded part reduces the vehicle speed to $10 \mathrm{~km} / \mathrm{h}$ [12]. This paper deals with the performance of the Nantes ring road under uncertainty and disruption crises, and the management of the potential forces (crises) to evaluate the performance of the ring road. The keys to crisis management are anticipation, planning, preparation, and training. Much of the responsibility for such action rests with the executive board of directors and the road secretary [5]. The crisis management framework studies the crisis in three stages, (i) Management Phases include a loop of pre-crisis (Preparedness), during the crisis (Response), and the post-crisis (Recovery and Mitigation). (ii) Interaction between crisis respondents (Public, Organizations), and (iii) Information Management Enablers (Information Collection, Communication, and Collaboration) [13]. Damage by flood hazards depends on the vulnerability of exposed elements. The term vulnerability refers to the inherent characteristics of these elements which determine their potential to be harmed [14]. Road traffic jams continue to remain a major problem in most cities around the world, especially in developing regions resulting in massive delays, increased fuel wastage, and monetary losses. Several metrics define traffic characteristics such as speed, flow, and density of a road [15].

The systematic combination of simulation methods with empirical research is a powerful tool in risk management research. This study considers the vehicles as agents which are circulating on the ring road so that it simulates the road traffic problem through Agent-Based Modeling (ABM). Most of the commonly used ABM platforms follow the "framework and library" paradigm, providing a framework - a set of standard concepts for designing and describing ABMs-along with a library of software implementing the framework and 
providing simulation tools [16]. There is some software to simulate the traffic considering the capacity of the roads, buffer size, exit rate, etc. (e.g. Aimsun (Transport Simulation Systems (TSS)), CUBE, Dynameq, MITSIMLab, Simtraffic, etc.) [17].

In this paper, quantitative agent-based and stochastic simulation modeling of the ring road is used as it best fits the complexity, context, and essence of the ring road with routes and agents (vehicles). Such simulation modeling is embedded into the physics-based crisis management framework to simulate the traffic process in the ring road and to assess and mitigate the potential crisis related to the ring road.

\section{Physic-based crisis management framework}

\subsection{General Perspective of POD for crisis management}

A system might be destabilized by unforeseen changes. These changes mainly refer to the variation of system parameters and consequently, deviation from the system's expected trajectory. The expected trajectory depends on the considered case, but this is often the targeted, planned, or most probable one. Any deviation from that trajectory is considered a crisis for the system. System attributes define the different situations of the system. Identifying these attributes, their relationship, and their level of security is required to design a smart predictor system. The Physics Of Decision (POD) framework introduced in section 1 defines two spaces in which a system can be positioned [9].

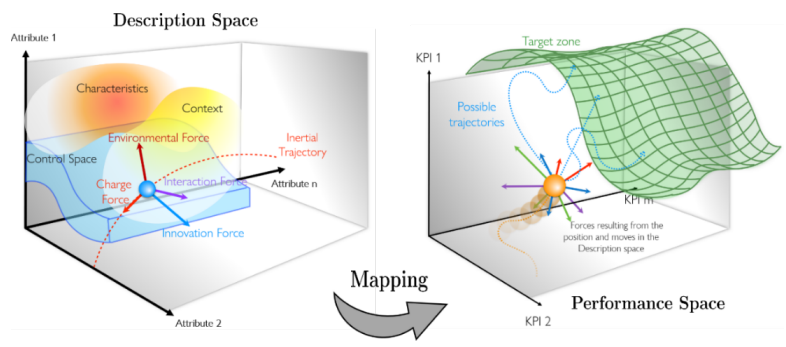

Figure 3: POD-based intelligent crisis management framework.

The Performance Space, depicted on the right side of Fig. 3, describes the performance of the system in a given state by locating it relative to KPIs, with crisis being shown as force vectors in that referential.

The Description Space, depicted on the left side of Fig. 3, represents the system's states by locating it relative to axes defined according to its attributes.
Some attributes are changed at certain times by decision-makers to control the deviation of trajectories. The degree of changes for each attribute is determined by system constraints in the Control Space, which is basically the "easy to access" subpart of the Description Space.

The relationship between the two spaces is determined by a function of Attribute(s) to KPI(s), which maps Description Space to Performance Space. This function can be straightforward or complex [8]. In cases where equations between the two spaces can be determined, it would be possible to analyze the data and deviations from the normal trajectory caused by the forces. Otherwise, this function should be determined differently, notably through simulation experiments as in this paper, so as to generate the data from which the unknown relationships between attributes and KPIs can be inferred [9].

\subsection{Problem statement}

The Interdepartmental Direction of Western Roads (DIRO) and Cofiroute operators are responsible for frequent floods on the northeast part of the Nantes ring road [2]. To cope with this crisis, the DIRO must close the ring road, partially or totally (depending on the extent of the submersion of the water to the roadway), between two stations, "Porte de la Chapelle" and "Porte de la Beaujoire" which are two ring road interchanges then they activate other routes (called: "S" alternative routes) which are major alternative adjacent routes in the flooded part [2, 3]. The DIRO decides to close the ring road (totally or partially) between the aforementioned ports based on the Gesvres water level reported by "La Jonelière", measuring station nearby the river according to the following indicator for the water level [3, 10]:

- $203 \mathrm{~cm}$ : water arrives on the right lane of the outer ring road;

- $210 \mathrm{~cm}$ : water arrives on the left lane of the outer ring road;

- $228 \mathrm{~cm}$ : water arrives on the left lane of the inner ring road;

- $230 \mathrm{~cm}$ : water arrives on the right lane of the inner ring road.

The reaction of the ring road operators to the crisis is critical in the flooded part and it gets increasingly more critical if the flood happens during peak hours usage of the ring road. This article mainly focuses on assessing the capability of the ring road to handle the traffic in normal days usage and the flooding days with different 
possible times of onset and intensity. The major focus of the case study is ascertaining the bottlenecks and rupture points in the Nantes ring road where issues may emerge from the perspective of the POD-based intelligent crisis management framework.

\section{Methodology}

\subsection{Nantes ring road architect and Gesvres flood}

The inner roadway of the Nantes ring road has 23 exits and 24 entrances and the outer roadway has 23 exits and 22 entrances (there is one exit for almost every entrance). Exceptions are located at interchanges 43 (Porte d'Anjou) on its inner roadway and interchange 33 (Porte d'Or Mor) on its outer roadway which have two entrances and two consecutive exits respectively (see Fig. 4).

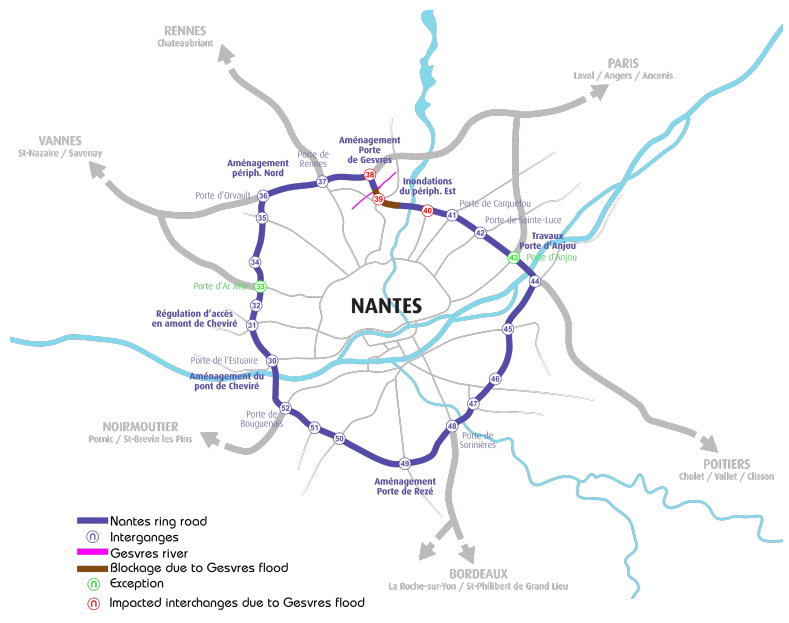

Figure 4: Nantes ring road map.

As soon as the water of the Gesvres river rises and reaches the minimum blockage indicators, the ring road is closed (partially or totally depending on the flood intensity) and the alternative routes are activated to prevent the traffic jam. The alternative route S6 is activated for the vehicles in the south-north ( $\mathrm{SN}$ ) direction (on the outer roadway) and S5 is activated for vehicles in the north-south (NS) direction (on the inner roadway). These two detour routes are indicated for ring road users by specific signs or messages before the blockage part [18].

According to the slope of the ring road in the blockage part, the outer roadway flooded first then based on the intensity of the flood, the water approaches the inner roadway. The two alternative routes S5 and S6

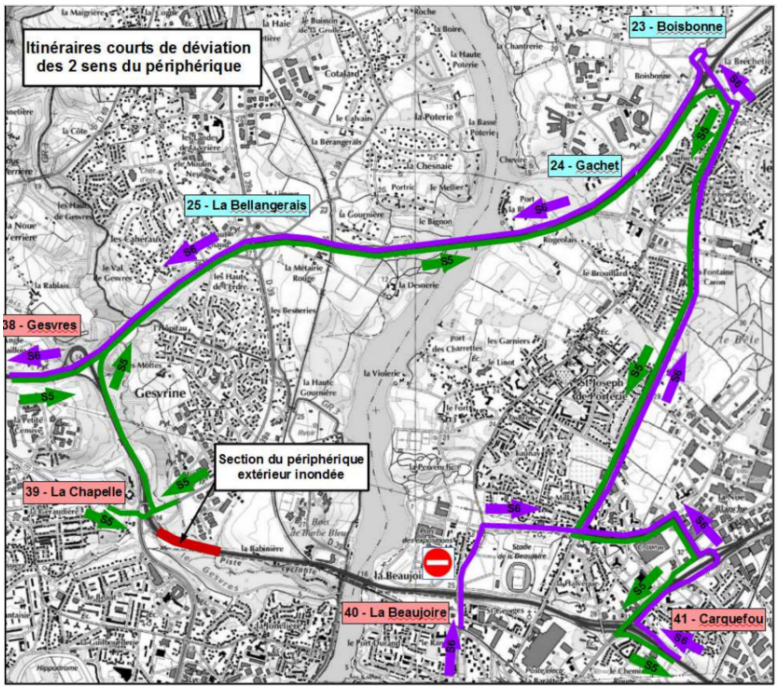

Figure 5: Alternative routes during Gesvres flood on the Nantes ring road (DIRO, 2015).

follow the urban network of Nantes Metropole and the Cofiroute motorway network.

\subsection{Traffic jam topology and its process modeling}

The traffic jam process is modeled to begin with the entry of a vehicle from one of the 46 entrances (inner and outer) to the Nantes ring road. Once on the ring road, the vehicles circulate on the ring road until they reach their destination. The simulation initializes at 12 midnight so that generally a small number of vehicles are traveling (of course after one full day running the simulation, some vehicles, oncoming vehicles, already travel on the ring road). To reflect the real traffic situation, the whole length of the ring road is segmented into dual segments among each two consecutive exists.

The first segment is from an exit to the first next entrance and the second segment starts from the first observed entrance to the next exit. This process is applied to the whole ring road (inner and outer roadways). This process correctly illustrates the logic of a moving vehicle that has passed an exit on the ring road (the vehicle definitely sees and passes the first next entrance and travels until the next exit and finally decides to exit or keeping continue on the ring road).

The next step is the traffic jam implementation. The vehicles that have passed the first exit, cannot enter segment 1 while there is no space (the minimum required space is at least the vehicle length). The same logic is applied for the vehicles that have passed segment 1 and are trying to enter segment 2. Besides, those 


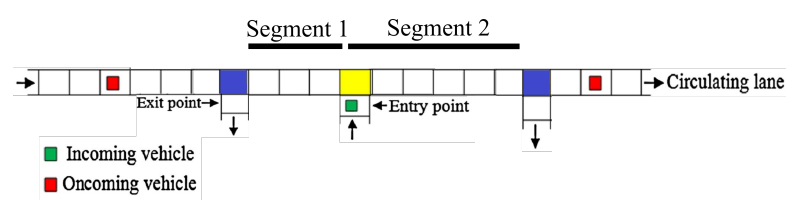

Figure 6: Traffic logic between two exits (including 2 segments) [19]

that are entering the ring road from entrances (Incoming vehicle (green) at yellow cell in Fig. 6) have to stop in the detour route leading to ring road until there is required space at segment 2 . The parameters of the implemented traffic process are given in section 4.4 . The traffic jam process is applied to the alternative routes (S5 and S6) as well.

Fig. 7 shows the schema of a sample of whole segmented routes on the Nantes ring road for implemented Traffic jam process with the Process Modeling and GIS libraries of the AnyLogic $(\odot)$ simulation software used in this study.

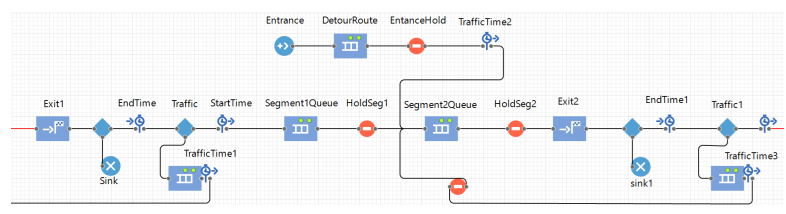

Figure 7: Traffic jam process modeling for the Nantes ring road.

AnyLogic $\odot$ Process Modeling Library supports discrete-event, or, to be more precise, process-centric modeling paradigm. Process Modeling Library objects can model the real-world systems in terms of agents (transactions, customers, products, parts, vehicles, etc.), processes (sequences of operations typically involving queues, delays, resource utilization), and resources. The processes are specified in the form of flowcharts - a widely adopted graphical representation used in many areas: manufacturing, call centers, business processes, logistics, healthcare, etc.

AnyLogic(@) flowcharts are hierarchical, scalable, extensible, and object-oriented, which enables the user to model large complex systems at any level of detail. Another important feature of the Process Modeling Library is the ability to create very sophisticated animations of process models. Besides, the GIS library uses the GIS map to implement the routing and navigation from any origin (point/region) to a destination. This library requests the routing from Open Street Map (OSM) server for considered routes with the possibility of shortest or fastest routes for any agent type (car, rail, bike, foot, etc.) [20].

\subsection{Safe driving modeling}

As long as there is no other vehicle in the sight of a moving vehicle, the vehicle will move at the freeway's speed limit (the paper considers other possible objects appearing in the path of a moving vehicle as another vehicle). The initial speed considered for the vehicles is a triangular distribution between the minimum and maximum speed limit. The considered initial speed for the ring road is:

\section{Triangular $(70 \mathrm{~km} / \mathrm{h}, 90 \mathrm{~km} / \mathrm{h}, 80 \mathrm{~km} / \mathrm{h})$}

These values may change when there are other vehicles in the sight of the moving vehicles' drivers. To simulate the process of decreasing and increasing speed on the ring road, the simulation applies the "Two-second rule". The two-second rule is a rule of thumb by which a driver may maintain a safe trailing distance at any speed [21, 22]. The rule is that a driver should ideally stay at least two seconds behind any vehicle that is directly in front of his or her vehicle. It is intended for automobiles, although its general principle applies to other types of vehicles. Depending on the distance between the moving vehicle and the newly appeared vehicle, the simulation decreases the speed of the vehicle until the vehicle is within the Two-second rule (likewise, the speed restore gradually as soon as the capacity is back to the allowable range). This process continues until the number of vehicles reaches the maximum capacity of the segment on which the vehicle is moving and as soon as the segment is full, the Hold markup is activated (see Fig. 7).

\subsection{Data sources and scenarios}

The simulation utilized various parameters as attributes in the Description Space. These parameters include the values of (i) the vehicles as agent (e.g. car, truck) includes their flows, length, origin, speed, and destination (ii) the ring road includes total length, segment's lengths, speed limit, capacity (one-line or more than one-line roadway), and (iii) the flood includes the time of onset (date and time) and its magnitude.

The Gesvres water level (as the magnitude of the flood) is considered as the origin of applied force to the system (section 3.2). The goal is then to evaluate the probable forces of the modeled crises and their impact on the system trajectory in the Performance Space of the Nantes ring road to reflect a realistic simulation of the traffic jam process.

This section gives more details about the aforementioned parameter considered as Attributes for the Description Space, in the other words, the simulation inputs. 
4.4.1. Agent The Description Space related to the parameters of the vehicle, ring road, and the river. This section is related to the parameters of the vehicles considered as agents.

Flow: The simulation only had access to data recorded by some on-road cameras (not all of them) include the flow of the vehicles passing them and their speed (Oncoming vehicles in Fig.6). Lack of access to the flow and speed of the vehicles at entrances and exits was a challenge. To distribute the entire flow of vehicles recorded by on-road cameras between entrances and exits, the two following possibilities are considered concerning the current position of a sample vehicle. (i) Possibility of departure of the intended vehicle while traveling on the road (Oncoming vehicle) at its upcoming exits, (ii) Possibility of departure of a newly arrived vehicle (Incoming vehicle in Fig. 6) on the ring road at the upcoming exits. The first hypothesis is to ensure the exit of an arrived vehicle on the ring road after crossing the maximum halfway of the ring road. Otherwise, the driver should have chosen the opposite direction (this hypothesis is based on heavy traffic data and the almost always optimal time selection of the shortest route). With these hypotheses and trial and error by varying the possible values for the exit probability through the simulation campaigns, the following formula has been obtained for the ring road exits. (U:Uniform, I: Incoming vehicles, O: Oncoming vehicles)

$\operatorname{Pr}\left(\right.$ exit $\left._{i}\right)=1-\frac{U(0.7,0.95) \times \Sigma I_{i}+U(0.4,0.6) \times \Sigma O_{i}}{\Sigma I_{i}+\Sigma O_{i}}$

According to Fig. 7, the exit probability at the second exit markup in the schema (the distinguisher connected to sink 1) is calculated for the vehicles between 'StartTime' and 'Exit2' markups and the vehicles between 'Entrance' and 'Exit2' markups.

The second issue with the vehicle flows was the lack of access to all on-road cameras. The simulation had access to the recorded data of 6 on-road cameras located between exit 40 (Porte de la Beaujoire) and 45 (Porte de Goulaine) (see Fig. 4). To feed the flows of entrances and exits in the simulation on the rest exits, the simulation is duplicated the existing data of exits 40-45 to other similar congestion exits. This simulation feed is implemented according to the vehicle congestion map shown in Fig. 8 concerning the high flow in the south-north direction in the morning and the opposite in the evening.

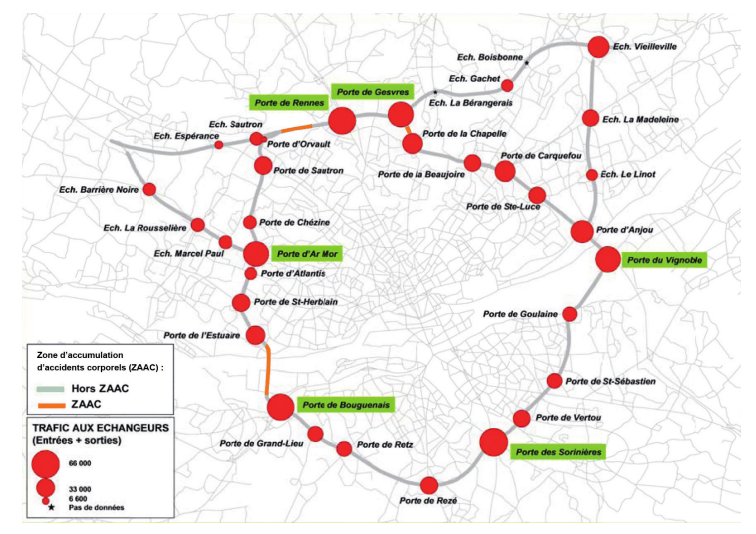

Figure 8: Nantes Agglomeration Traffic Operating System (DREAL - 2010).

Length: The average length of vehicles in terms of type, e.g. car or truck, uniform $(4.5,5.5)$ meter has been considered [23]. Besides, the minimum considered distance between the agent in heavy traffic is 1 meter.

4.4.2. Ring road Section 4.1 is dedicated to the Nantes ring road architect and its interchanges. In this section, the considered values for the ring road as another attribute in Description Space are explained.

Length and capacity: The Nantes ring road is $43 \mathrm{~km}$ long. The total length is segmented into dual segments. The first segment start from an exit to the first next entrance and the second segment starts from the first observed entrance and ends at the next exit (see Fig. 6). To calculate the capacity of each segment, the total length of the segment in meters (automatically is calculated by the OSM server) concerning the minimum distance between two consecutive agents has been divided by the average length of the vehicles.

Speed limit: The permitted speeds were selected to corroborate the rule is used to determine traffic jams in the main cities in France. These rule judges that, for a regulated speed between $70 \mathrm{~km} / \mathrm{h}$ and $90 \mathrm{~km} / \mathrm{h}$ and at maximum $60 \mathrm{~km} / \mathrm{h}$ for the alternative routes. Besides, the following indicator has been considered for the traffic status:

- Traffic is fluid if the speed is greater than $50 \mathrm{~km} / \mathrm{h}$,

- Traffic is dense if the speed is between $30 \mathrm{~km} / \mathrm{h}$ and $50 \mathrm{~km} / \mathrm{h}$,

- Traffic is congested if the speed is less than 30 $\mathrm{km} / \mathrm{h}$ 
4.4.3. Flood There are different parameters related to the flooding of the Gesvres river. Some of these parameters are precipitation, rainfall intensity, humidity index, slope, land use, etc. The only parameter considered in this study is the Gesvres water level reported by "La Jonelière" measuring station nearby the river and disrupt the system according to the defined indicator in section 3.2

The attributes' values can vary in some specific ranges because of the degrees of liberty for the ring road considering, capacity, speed limits. These variations are considered in the control space of Description Space (Fig. 3). Besides, the water level of Gesvres can vary and impacts the ring road performance. The water level is out of control and is considered as "Characteristic" in the POD framework. The water level's impact on ring road performance (fluid, dense, and congestion traffic) is considered as "Environmental Force" in Fig.3.

According to the objectives of the ring road, these variations impact the ring road's performance. To evaluate the ring road performance, the following Key Performance Indicators (KPIs) are considered:

- The average speed of all exits for each direction (inner and outer roadway of the ring road) and the GIS points on the alternative routes,

- The average flow of vehicles on the ring road and the alternative routes,

- The Average Trip Time (ATT) between interchange 37 and interchange 44 as critical path that could be impacted by the Gesvres flood.

\subsection{Disruption Scenarios}

Some possible scenarios are considered to implement in the simulation and study their impact on the system's trajectories in the performance space. These scenarios mainly refer to the flood attributes. Table 1 provides illustrative distinct possible Disruption Scenarios (DS) for the traffic on the Nantes ring road.

Table 1 shows a very limited number of possible disruption scenarios. These scenarios considering the onset of the flood, its intensity, and the interval of the flood (start-end period of the flood) could be different. Table 1 in case of flood, considers the possible disturbances from the beginning of the flood to its end on the same day.
Table 1: Possible Disruption Scenarios (DS) for the Gesvres flood on the Nantes ring road.

\begin{tabular}{llrr} 
DS & Description & Onset & Magnitude \\
\hline DS1 & $\begin{array}{l}\text { Partial flood at peak } \\
\text { hour }\end{array}$ & $08: 30 \mathrm{am}$ & $207 \mathrm{~cm}$ \\
\hline DS2 & $\begin{array}{l}\text { Partial flood at peak } \\
\text { hour }\end{array}$ & $08: 30 \mathrm{am}$ & $215 \mathrm{~cm}$ \\
\hline DS3 & $\begin{array}{l}\text { Total flood at normal } \\
\text { hour }\end{array}$ & $12: 00 \mathrm{pm}$ & $240 \mathrm{~cm}$ \\
\hline DS4 & $\begin{array}{l}\text { Total flood at peak } \\
\text { hour }\end{array}$ & $08: 30 \mathrm{am}$ & $240 \mathrm{~cm}$ \\
\hline
\end{tabular}

\section{Experiment and results}

The main experiment is centered on the crisis-affected performance of the Nantes ring road. The heavy traffic jam of the ring road due to the Gesvres flood in its northeast part is considered a crisis which is the physical force in the POD framework. In the perspective of the physics-based crisis framework, deviations from the inertia trajectory (i.e. the most probable scenario) are considered as perturbation forces.

According to the POD framework, the first and foremost trajectory is the inertia trajectory which is the reference trajectory to study different possible scenarios. The flow of the vehicles, the capacity of the ring road (i.e. the segments' capacities), and the limit speed of the ring road are considered to track the inertia trajectory of the ring road. The considered flow for the simulation is for February $5^{\text {th }}$ of 2020 (Wednesday, a typical working day in France).

The result related to the inertia trajectory is for February $5^{\text {th }}$, 2020. The values for the ring road capacity calculated by the OSM server through AnyLogic $($ software and the triangular distribution for the limit speed in section 4.3, is considered. The simulation results for the "Inertia" trajectory is shown with magenta color in Fig. 9.

Three disruption scenarios are investigated for the Nantes ring road in terms of flood parameters. Scenarios are related to the onset time and magnitude of the flood at Gesvres river (Table 1). But they could be distinguished scenarios only related to the onset or only related to the magnitude.

The results for the considered scenarios (Table 1) are shown in Fig. 9. The results are for the following interval on $5^{\text {th }}$ of February 2020: (00:00:00-23:59:59). The results for the inertia indicate the high flow from south to north in the outer ring in comparison to the inner 

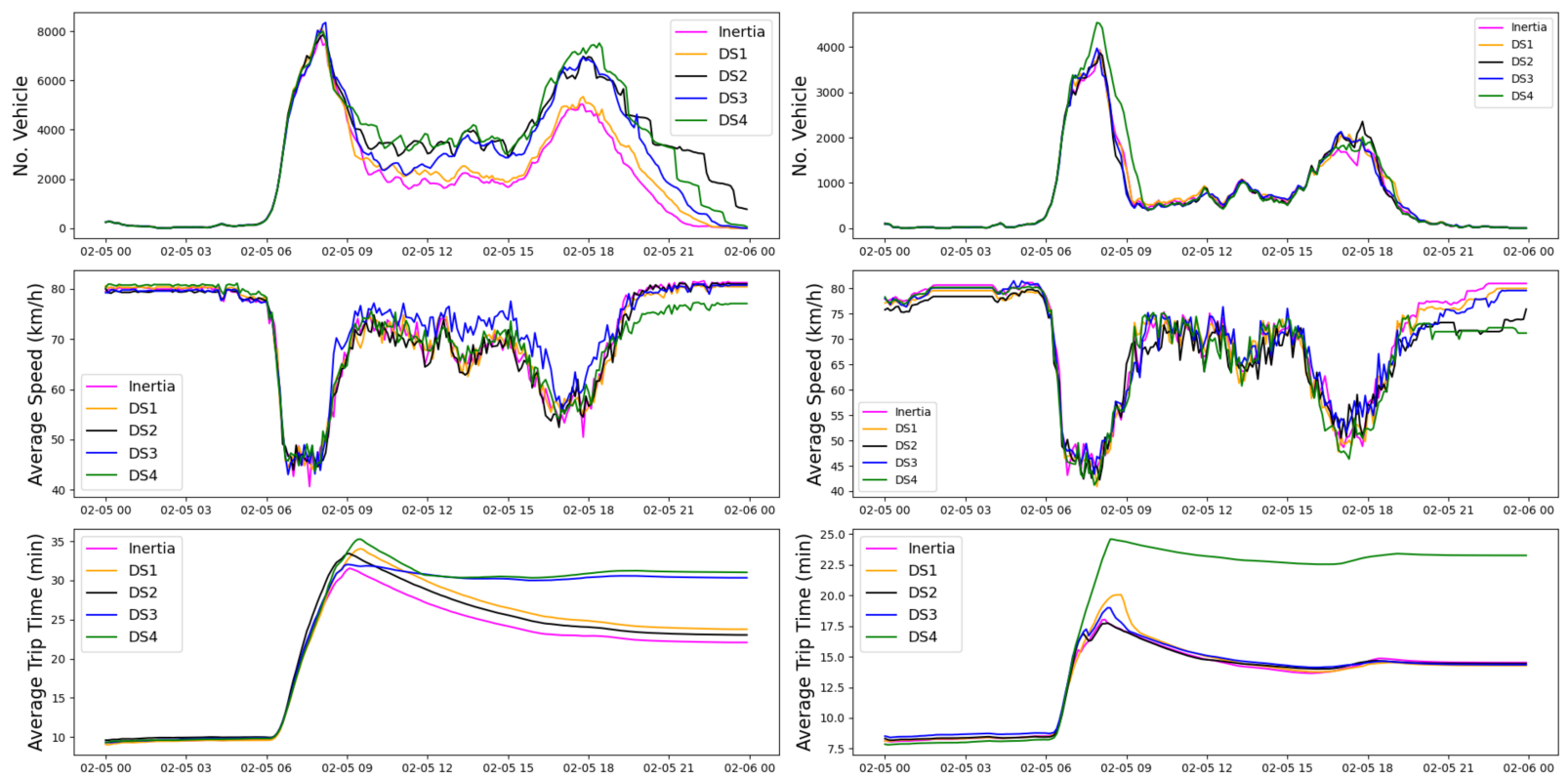

Figure 9: The simulation results for the Nantes ring road and its possible crises.

ring due to the high number of jobs located in the north on the Nantes city. The average speed for the inertia is close to the minimum of the triangular distribution for the limit speed which means the two-line roadway of the Nantes ring road is not quite enough for the vehicle's flow on a normal day. The flow of vehicles on the outer roadway is definitely impacted by DS2, DS3, and DS4. These results show the importance of the onset and magnitude of the flood on the ring road performance. The selected distance between interchanges $(37,44)$ is about $12.5 \mathrm{~km}$. Considering the limit speed for the ring road, this distance ideally should take 8-9 minutes to travel. The Average Trip Time in "Inertia", "DS3", and "DS4", highlights the following conclusions respectively: (1) lack of road lines for the most probable vehicle flow, (2) the high impact of the Gesvres flood in the outer ring of the ring road and the low capacity of the alternative routes chosen to control the traffic, and (3) besides (1) and (2), the alternative routes (S5 and S6) have a very low capacity to handle the traffic caused by the river flood in the inner roadway.

The performance space gives an overview of the KPIs to the decision-maker while it would be more helpful to have a comparative image of the Nantes ring road traffic-oriented performance. To reach this objective, the KPIs should be examined on the same scale in the performance space. The performance spaces in Fig. 10 are presented for the following KPIs for the inner and outer roadway of the Nantes ring road in two different performance spaces and their comparisons with non-flooding day (inertia trajectory): (i) Vehicle flow, (ii) Average speed on the roadway, and (iii) Average Trip Time between the two critical interchanges on the ring road.

\section{Conclusion and perspectives}

This article presented the application of the Physics Of Decision (POD) approach to the crisis situations. The empirical experimentation reported in this paper assesses and quantifies the impact of disruptions on a sensitive highway to heavy traffic jam due to blockage in its specific part. This evaluation is essentially based on an agent-based simulation model, fed on the one hand by vehicle flow forecasts and the structural features of the road and on the other hand by the description of the consequences of disruptions on the system's parameters. The obtained results mainly concern the visualization of performance trajectories and the deviations generated by the anticipated disturbances. The dynamic simulation of the water level in the flooded part of the ring road and connecting it to the current simulation could be the next step for the presented work.

One important takeaway from the presented work in this article concerns the use of forecasts: The next step is to show how real-time data could be used directly to benefit from the presented contributions and provide a live management dashboard to control the traffic jam on a road exposed to blockage. The decision-makers could then visualize or monitor the performance of the 


\section{Performance Space of Outer roadway}

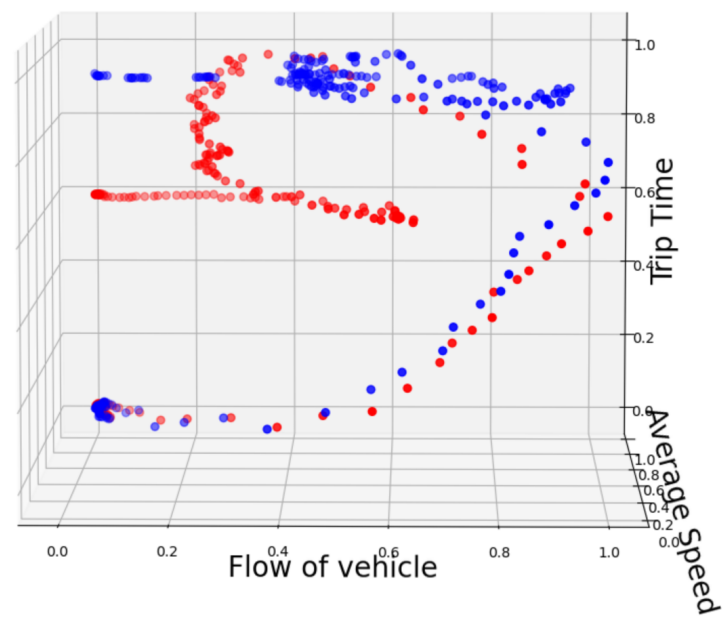

\section{Performance Space of Inner roadway}

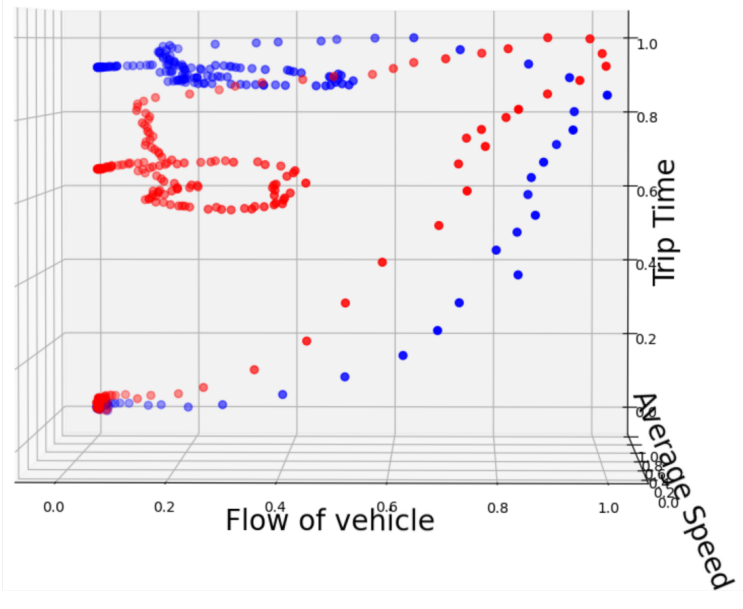

Figure 10: Scaled Inertia trajectories (red trajectories) for outer and inner roadway vs Scaled deviated trajectories (blue trajectories) for the Nantes ring road exposed to Gesvres river.

system live and anticipate the impact of current or future disruption to support their decision-making process.

\section{References}

[1] Wikipedia, "Boulevard périphérique de nantes." https://fr.wikipedia.org/wiki/

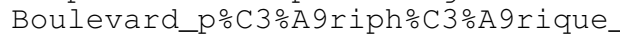
de_Nantes, Jun 2021.

[2] D. i. d. r. Ouest, "Interdepartmental directorate of west roads." http://www.dir.ouest. developpement-durable.gouv.fr/. Jun 2021.

[3] D. P. d. 1. Loire, "Regional directorate for the environment, planning and housing, rn844 périphérique nantais - enjeux et projets." http://www.pays-de-la-loire. developpement-durable.gouv.fr/, Sep 2018.

[4] Anonym, "The northern ring road again cut by the gesvres flood, the roadway is flooded between the porte de la beaujoire and the porte de la chapelle," Mar 2020.

[5] J. KERISEL and H. GONIN, "The crossing the marais de gesvres by the north rocade of the city of nantes," REV GEN ROUTES AERODR, no. 543, 1978.

[6] G. Macdonald and K. Macdonald, "Safeguarding: A case for intelligent risk management," British Journal of Social Work, vol. 40, no. 4, pp. 1174-1191, 2010.

[7] F. Benaben, B. Montreuil, L. Faugere, M. Lauras, J. Gou, and W. Mu, "A physics-based theory to navigate across risks and opportunities tn the performance space: application to crisis management," in Proceedings of the 53rd Hawaii International Conference on System Sciences, 2020.

[8] N. Moradkhani, L. Faugère, J. Jeany, M. Lauras, B. Montreuil, and F. Benaben, "A physics-based enterprise modeling approach for risks and opportunities management," in IFIP Working Conference on The Practice of Enterprise Modeling, pp. 339-348, Springer, 2020.

[9] F. Benaben, L. Faugere, B. Montreuil, M. Lauras, N. Moradkhani, T. Cerabona, J. Gou, and W. Mu, "Instability is the norm! a physics-based theory to navigate among risks and opportunities," Enterprise Information Systems, pp. 1-28, 2021.

[10] J. Urbach, "Point noir du périph nantais, la porte de gesvres bientôt transformée," Oct 2020.

[11] "Jonelière station for the gesvres water level."

[12] Anonym, "Traffic difficulties on the nantes ring road, which is partly flooded - tellerreport.com."

[13] N.-S. Lee, S. Hirschmeier, S. Müller, and L. J. Luz, "Enablers in crisis information management: a literature review," in Proceedings of the 50th Hawaii International Conference on System Sciences, 2017.

[14] J. Schanze, "Flood risk management-a basic framework," in Flood risk management: Hazards, vulnerability and mitigation measures, pp. 1-20, Springer, 2006.

[15] V. Jain, A. Sharma, and L. Subramanian, "Road traffic congestion in the developing world," in Proceedings of the 2nd ACM Symposium on Computing for Development, pp. 1-10, 2012.

[16] E. Kiesling, M. Günther, C. Stummer, and L. M. Wakolbinger, "Agent-based simulation of innovation diffusion: a review," Central European Journal of Operations Research, vol. 20, no. 2, pp. 183-230, 2012.

[17] E. B. Lieberman, "Brief history of traffic simulation," Traffic and Transportation Simulation, vol. 17, 2014.

[18] R. Actu, "Inondations : une partie du périphérique de nantes fermée, la situation ne s'améliore pas," Mar 2020.

[19] H. Echab, N. Lakouari, H. Ez-Zahraouy, and A. Benyoussef, "Phase diagram of a single lane roundabout," Physics Letters A, vol. 380, no. 9, pp. 992-997, 2016. 
[20] "Anylogic simulation software, process modeling library."

[21] "Two-second driving rule." https://en. wikipedia.org/wiki/Two-second_rule, Jan 2021.

[22] D. Uribe, "Multi-agent approach to the two-second driving rule," Journal of Computers, p. 168-175, 2018.

[23] Magnus.sellen, "Average car length [list of car lengths by car type]." https://mechanicbase.com/cars/ average-car-length/Feb 2021. 